\title{
The effects of enactment and intention accessibility on prospective memory performance
}

\author{
Janette C. Schult ${ }^{1} \cdot$ Melanie C. Steffens ${ }^{1}$
}

Published online: 15 December 2016

(C) Psychonomic Society, Inc. 2016

\begin{abstract}
The intention-superiority effect denotes faster response latencies to stimuli linked with a prospective memory task compared to stimuli linked with no prospective task or with a cancelled task. It is generally assumed that the increased accessibility of intention-related materials contributes to successful execution of prospective memory tasks at an appropriate opportunity. In two experiments we investigated the relationship between the intention-superiority effect and actual prospective memory performance under relatively realistic conditions. We also manipulated enactment versus observation encoding to further investigate the similarity in representations of enacted and to-be-enacted tasks. Additionally, Experiment 1 included a control condition to investigate the development of the intention-superiority effect over time. Participants were asked to perform prospective tasks at the end of the experiment to prepare the room for the next participant. They studied these preparatory tasks at the beginning of the experiment either by enacting them themselves or by observing the experimenter perform them. In Experiment 2, participants in a control condition did not intend to perform prospective tasks. We observed a smaller intention-superiority effect after enactment encoding than after observation encoding, but only if response latencies were assessed immediately before the prospective memory task. In addition, Experiment 2 suggested that the size of the intentionsuperiority effect is related to successful prospective memory
\end{abstract}

Melanie C. Steffens

steffens@uni-landau.de

1 Department of Social, Environmental, and Economic Psychology, Faculty of Psychology, University of Koblenz-Landau,

Landau, Germany performance, thus providing evidence for a functional relationship between accessibility and memory.

Keywords Prospective memory $\cdot$ Intentions $\cdot$ Enactment . Observation $\cdot$ Intention-superiority effect

A fascinating feature of human memory is the ability to realize previously planned intentions after a delay interval at an appropriate moment: prospective memory. A key feature of many prospective memory tasks (in contrast to retrospective memory tasks) is the absence of an explicit reminder to recall the task when an opportunity to realize the intention occurs (e.g., Graf \& Uttl, 2001). For example, if you drank the last drop of milk this morning, no one would remind you on your way home from work to stop at the supermarket to buy some more. An important question is which processes trigger the successful detection of a retrieval opportunity (i.e., the supermarket) and the retrieval of the associated activity (i.e., buy milk; for formal models of prospective memory, see Gilbert, Hadjipavlou, \& Raoelison, 2013; Horn, Bayen, Smith, \& Boywitt, 2011). The aim of this research is to test how the representation of intentions during the delay interval affects prospective memory performance.

The intention-superiority effect is the finding that stimuli associated with an intention trigger a response faster than do stimuli associated with no intention or with a cancelled intention. In two experiments, we tested whether this intentionsuperiority effect contributes to successful prospective memory performance (i.e., the self-initiated realization of delayed intentions at an appropriate moment). Concurrently, we further investigated the functional similarity in the representations of tasks that have been carried out and tasks one intends to carry out later (i.e., between enacted and to-be-enacted tasks). Finally, Experiment 1 further investigated the question 
whether intention-related memory content remains activated throughout a delay interval, or not, and Experiment 2 included a control condition in which participants did not intend to perform prospective tasks.

\section{The intention-superiority effect}

How can the cognitive representation of intentions be operationalized in controlled experiments? To answer this question, Goschke and Kuhl (1993) introduced the postponed-intention paradigm. Typically, participants study two short lists of actions. Afterward, they are asked to enact one script (prospective list), but not the other one (control list). Importantly, a short delay task is introduced first. During this delay, response latencies for stimuli of both lists are assessed. Faster response latencies for words on the prospective list than words on the control list were demonstrated in recognition memory tests (e.g., Goschke \& Kuhl, 1993; Schult \& Steffens, 2013) and lexical decision tasks (e.g., Dockree \& Ellis, 2001; Marsh, Hicks, \& Bink, 1998; Marsh, Hicks, \& Bryan, 1999; Penningroth, 2011; Penningroth, Graf, \& Gray, 2012; Schult \& Steffens, 2011). Goschke and Kuhl (1993) interpreted the intention-superiority effect in terms of increased accessibility of intention-related content compared to other memory content (e.g., Ellis, 1996; Förster, Liberman, \& Higgins, 2005; Lewin, 1926, for a similar reasoning). They suggested that intentions are represented as special source nodes that decay more slowly than neutral content. A more persistent activation could improve the likelihood of recollections of the intention during the delay interval, which, in turn, should increase the strength of activation of the representation and could initiate more strategic monitoring of an appropriate opportunity to realize the intention (e.g., Ellis, 1996; Kvavilashvili \& Fisher, 2007). As an alternative explanation, McDaniel and colleagues proposed that the unexpected ease of processing intention-related information relative to other information could also spontaneously trigger search processes. That is, people try to find a viable source to account for differences in processing quality (e.g., Lee \& McDaniel, 2013; McDaniel, Guynn, Einstein, \& Breneiser, 2004, for a more detailed discussion of the discrepancy-plus-search hypothesis). Put simply, people ask themselves why the intention-related information was processed so easily, and this may remind them of their intention. Thus, successful prospective memory performance should be more likely if people attribute the increased accessibility of information to the intention.

We previously found an intention-superiority effect in a recognition test, but only when participants expected to execute these intentions immediately after the recognition test. If they did not expect to execute the intentions later, there was no effect (Schult \& Steffens, 2013). In other words, the intentionsuperiority effect is not observed throughout the retention interval, but only immediately after intention formation and then again when a retrieval opportunity is near.

\section{Prospective memory after enactment encoding}

Freeman and Ellis established another possible reason for the increased accessibility of information in a postponed-intention paradigm (Freeman \& Ellis, 2003a, 2003b). They proposed that the intention-superiority effect is based on the activation of motor or sensorimotor information associated with the future enactment of an intention (see also Koriat, Ben-Zur, \& Nussbaum, 1990). In other words, materials should be more easily accessible both if they were enacted in the past or if they were intended for future enactment. To test their actionsuperiority account, Freeman and Ellis (2003b) asked participants either to enact or to read verb lists during the study phase and to form the intention to enact or to verbally recall the lists after a recognition test. They demonstrated faster response latencies for enacted as well as to-be-enacted verbs compared to a verbal-only control condition. Indeed, recognition latencies for enacted and to-be-enacted materials were comparable, and there was no intention-superiority effect for once enacted verbs. If materials were verbally encoded, to-be-enacted materials (i.e., the prospective list) were recognized faster than tobe-recalled materials (neutral list), replicating Goschke and Kuhl (1993). If, however, materials were already enacted during encoding, there was no difference in response latencies between the prospective and the neutral list, indicating functional similarities between enactment and the intention to enact. To the degree that recognition latencies reflect the ease of information processing, there seems to be no difference in processing quality for enacted and to-be-enacted information. In terms of the discrepancy-plus-search model (Lee \& McDaniel, 2013), such similarities could lead to more prospective memory failures for a previously executed task because easier processing is falsely attributed to previous enactment rather than the intention to execute such a task again.

In line with this reasoning, Schaefer and colleagues reported poorer prospective memory performance for participants who practiced the prospective tasks at the beginning of the experiment compared to participants who were just verbally instructed or who watched the experimenter demonstrate these tasks (Schaefer, Kozak, \& Sagness, 1998). Interestingly, all participants recalled at least some tasks when prompted (i.e., they showed retrospective memory), and mean recall rates were similar across encoding conditions. Therefore, differences in prospective memory performance were not based on impaired retention of the prospective tasks. The authors discuss several explanations for their finding of impaired prospective memory after enactment encoding. First, participants in the enactment condition could have underestimated the difficulty to remember the prospective task. Carrying out the 
simple preparatory tasks such as to sharpen a pencil could elicit a (false) feeling of competence, causing participants to spend fewer resources on the encoding or maintaining of the prospective task (for respective evidence, see von Stülpnagel, Schult, Richter, \& Steffens, 2016). Alternatively, carrying out the future preparatory tasks at the beginning of the experiment may have drawn attention to action-related information (what to do) at the cost of elaborating the relation between the appropriate retrieval opportunity and the action (when to perform the action). The high proportion of participants in the enactment condition who completely forgot the prospective task, but showed good retrospective memory, suggests that prospective memory failure was due to a missing cue for action initiation (i.e., the end of the experiment) rather than participants simply forgetting what to do. A third explanation could be that enactment encoding impairs the discrimination between enacted and to-be-enacted intentions. Freeman and Ellis's (2003b) demonstration of comparable response latencies for enacted and to-be-enacted verbs could reflect such difficulties in source discrimination. Research on enactment and source memory provides indirect evidence of this assumption. Source memory research suggests that the greater the overlap of information between two conditions, the more source confusions occur (e.g., Carroll, Mazzoni, Andrews, \& Pocock, 1999; Garry, Manning, Loftus, \& Sherman, 1996; Hornstein \& Mulligan, 2004; Manzi \& Nigro, 2008).

Several studies found a beneficial effect of enactment encoding on prospective memory, but in those studies the encoding task drew attention to the processing of the relationship between the intended action and the cue component (McDaniel \& Scullin, 2010, Exp. 3; Pereira, Ellis, \& Freeman, 2012a, 2012b). However, Schaefer et al. (1998), as well as the present research, focused on enactment encoding as a strategy to improve the retention of several intended activities, not as a strategy to strengthen the cue-action association. Therefore, we expected to replicate the pattern of findings Schaefer et al. reported. For retrospective memory, most studies have shown better memory after enactment encoding than other encoding conditions (e.g., Zimmer et al., 2001, for a review on the enactment effect in retrospective memory). However, participants who observe another person enact (i.e., observation encoding) typically show similarly good free recall as those enacting, but worse recognition (for a review, see Steffens, von Stülpnagel, \& Schult, 2015).

In a nutshell, previous studies have shown that enactment encoding has reduced both the intention-superiority effect and prospective memory performance, but not retrospective memory performance. This pattern of findings could be taken as evidence of the postulated functional relationship between the increased accessibility of intention-related content and prospective memory performance (Goschke \& Kuhl, 1993).

\section{The intention-superiority effect and prospective memory performance}

To the best of our knowledge, there are no published studies using the postponed-intention paradigm to directly link the intention-superiority effect with prospective memory performance. Because the typical postponed-intention paradigm does not involve prospective memory failure, this is not surprising. For example, the prospective and the neutral list are usually presented repeatedly during study (Goschke \& Kuhl, 1993; Schult \& Steffens, 2011, 2013), sometimes until both lists can be recalled perfectly (e.g., Marsh et al., 1998; Marsh et al., 1999; Penningroth, 2011; Penningroth et al., 2012; for an exception, see Dockree \& Ellis, 2001). Also, the lists typically consist of four to six related simple verb-object phrases describing a familiar script, such as tidying up a desk (but see Marsh et al., 1999, for a demonstration of an intention-superiority effect with lists of unrelated actions). Forgetting one or more actions of such lists during a delay interval lasting only a few minutes is rather unlikely. Consequently, reported recall performance is often perfect. It is also debatable whether the shortly postponed execution of a list of actions is equivalent to a prospective memory task that requires participants to remember to initiate the intended task at an appropriate moment on their own. In most studies using a postponed-intention paradigm it is unclear whether participants expected a prompt from the experimenter to initiate the execution of the postponed (prospective) task, or not. In addition, the short delay interval could encourage them to maintain the intention active in working memory (Lebiere \& Lee, 2002; Marsh et al., 1998).

Dockree and Ellis (2001) investigated the intentionsuperiority effect under conditions more similar to a relatively realistic prospective memory task. Adapting Schaefer et al.'s (1998) setting they asked their participants to clean up the desk for the next participant before leaving the room. During the experiment they cancelled half of the clean-up tasks (cancelled tasks; similar to the control lists used by Goschke \& Kuhl, 1993), but not the other half (prospective tasks), and assessed response latencies in a lexical-decision task (LDT) immediately after these instructions. Participants had to remember on their own that they were to carry out the prospective tasks about 8 minutes after the LDT. There was an intention-superiority effect in the LDT if participants expected to remember the prospective task themselves, but not if they expected a future reminder (i.e., a list describing all to-berealized tasks). Yet because prospective memory performance was perfect, the relation between intention superiority and prospective memory performance could not be tested.

In sum, research on the intention-superiority effect has demonstrated increased accessibility of stimuli associated with future enactment (i.e., faster reactions) compared to 
stimuli associated with no or cancelled enactment. The reaction-time difference decreased when participants enacted the intended activities during study (Freeman \& Ellis, 2003b). One drawback of most studies is that they do not contain a proper prospective memory task. Enactment at encoding impaired prospective memory in the same setting (Schaefer et al., 1998). Thus, if the high accessibility of intention-related content has a functional impact on prospective memory performance, smaller intention-superiority effects after enactment encoding should be linked with poorer prospective memory performance.

This article reports all data that we collected in order to test this idea (i.e., all experiments, conditions, and variables). Similarly, all statistical analyses that we computed are reported.

\section{Experiment 1}

Experiment 1 had two aims. First, we tested whether our instructions were sufficient to elicit an intention-superiority effect in a lexical-decision task and still produce failures in prospective memory performance. Similar to Schaefer et al. (1998) and Dockree and Ellis (2001), we asked participants to clean up before leaving the room at the end of the experiment (e.g., put the crayons back into the tin box). Following the typical paradigm to avoid confounds (Dockree \& Ellis, 2001), half of the prospective clean-up tasks were cancelled during the experiment, whereas the other half remained intention relevant.

Our second aim was to test for an intention-superiority effect at two different time points during the experiment. To gain further support for the context sensitivity of the intentionsuperiority effect (Schult \& Steffens, 2013), we manipulated the timing of the critical lexical-decision task. Each participant responded to a lexical-decision task in the middle of the experiment and to another one at the end. We expected to find the intention-superiority effect at the end of the experiment only.

We went to great lengths to mirror relatively realistic conditions in the laboratory and to discourage intentional memory strategies such as rehearsal. Specifically, and in contrast to previous studies, our participants did not know during encoding that the clean-up tasks would be important at a later point in the experiment. Also, in contrast to previous studies using a postponed-intention paradigm with reaction-time measurement, participants received no written instructions, but all tasks were explained orally, which made them less conspicuous. To investigate the relationship between enactment encoding, the intention-superiority effect, and prospective memory performance, participants either executed all cleanup tasks during instruction (enactment condition) or they watched the experimenter execute them (observation condition). We used an observation condition instead of a verbal control because both encoding conditions draw attention to action-related information and are thus more similar to each other than an enactment and a verbal control condition are. Figure 1 presents an overview of the procedure. Importantly, there was an extended instruction, practice, and planning phase that ended with intention formation (Phase 1). Afterward, participants carried out the activities that the cover story referred to, and the dependent variables were measured (Phase 2: lexical-decision task, prospective and retrospective memory tasks).

We tested the following hypotheses:

1. Participants in the enactment condition show poorer prospective memory performance than participants in the observation condition.

2. There is an intention-superiority effect for materials associated with the prospective tasks as compared to the cancelled tasks if participants expect to enact the clean-up tasks after the final lexical-decision task, but not if the critical lexical-decision task is administered in the middle of the experiment (when the prospective tasks are irrelevant).

3. If motor information decreases the intention-superiority effect, as predicted by the action-superiority account, in the (final) lexical-decision task we will observe a smaller reaction-time difference between prospective and

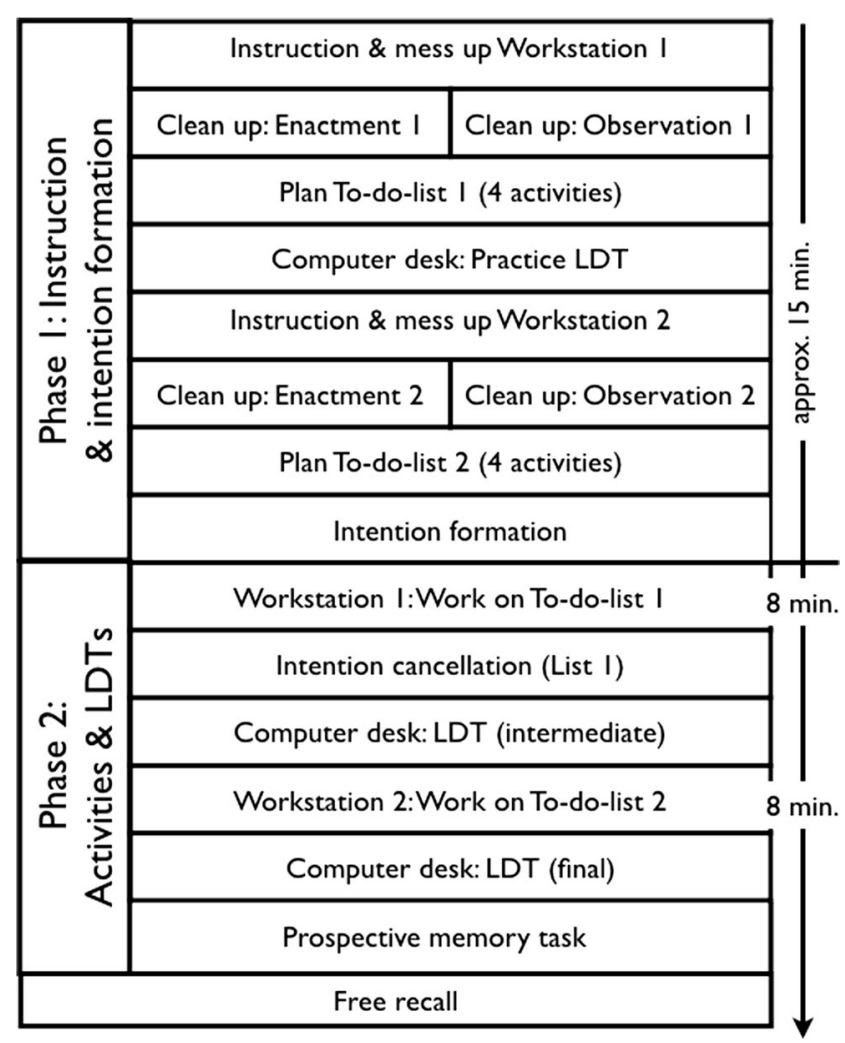

Fig. 1 Illustration of the procedure of Experiment 1 
cancelled tasks in the enactment condition than in the observation condition.

4. In a free recall task (retrospective memory), there should be no difference between scores obtained in the enactment and in the observation condition, whereas enactment should lead to better recognition than observation.

5. The intention-superiority effect should be related to prospective memory performance.

\section{Method}

\section{Participants and design}

A group of 52 students $\left(M_{\text {age }}=22.46\right.$ years, age range: $18-$ 42 years; $84 \%$ women) volunteered in exchange for course credit or a small payment. The design was a 2 (encoding condition: enactment vs. observation, between subjects) $\times 2$ (critical LDT position: intermediate vs. final, between subjects) $\times 2$ (stimulus type: prospective tasks, cancelled tasks, within subject) factorial. One participant (Observation $\times$ Final Condition) was excluded because she could not remember the prospective task at all (i.e., to clean up the desk), leaving 15 participants in the enactment + final condition, 14 participants in the observation + final, 10 participants in the enactment + intermediate, and 12 participants in the observation + intermediate condition. Sample size was determined in advance to obtain a statistical power of $1-\beta=.80$ to detect a large interaction effect ( $f=.40$; see J. Cohen, 1977) between encoding condition and critical LDT position in an ANOVA with $\alpha=.05$ (Faul, Erdfelder, Lang, \& Buchner, 2007). We expected at a large effect in Experiment 1 because it was uncertain whether the (expensive and time-consuming) procedure elicits both an intention-superiority effect in a lexicaldecision task and failures in prospective memory performance, which are the preconditions for testing Hypothesis 5.

\section{Materials}

Following Schaefer and colleagues (1998), we designed eight simple activities that used objects necessary for the prospective tasks. (Table 3 in the Appendix provides English descriptions of all German materials used.) For example, for the activity "draw a person," participants were instructed to use the crayons from a tin box. The corresponding prospective task was to put all used crayons back into the tin box before leaving the laboratory. Two material sets were constructed, each comprising four activities. The critical lexical-decision task (LDT) was modeled after previous studies (e.g., Dockree \& Ellis, 2001; Schult \& Steffens, 2011). It included the 16 nouns from the eight clean-up tasks that were counterbalanced (eight nouns referring to the cancelled tasks, eight referring to the prospective tasks), 16 new nouns, and 32 pronounceable nonwords. Words were comparable in word frequency class (Deutscher Wortschatz, 2016) (Task Set 1: $M=8.6, S D=2.4$; Task Set 2: $M=10.4, S D=2.5$, new words: $M=8.6, S D=2.0$, $F<2, p=.16$, Bonferroni-corrected pairwise comparisons: all $p s>.2$ ) and word length (Task Set 1: $M=17.9$ letters, $S D=$ 3.2, Task Set 2: $M=17.1, S D=2.6$, new words: $M=17.4, S D$ $=1.9, F<1)$. Nonwords were matched on word length with words. A second LDT with 32 activity-irrelevant nouns and 32 non-words was constructed. Another four stimuli (two words, two nonwords) served as buffers before each LDT.

\section{Procedure}

Each participant was tested individually by one of three female experimenters who had been trained extensively in lab rehearsals and learned detailed instruction scripts. All experimenters were unaware of the hypotheses. We used two rooms. Upon arrival, participants were greeted in Room A. The cover story that participants were told detailed the notion that we were interested in their ability to organize various activities as well as possible within a restricted timeframe. Their timemanagement skills would be tested twice, with four different activities each time. Their main task would be to gain as many points as possible; for some activities they would get points for correct answers, for other activities their creativity would be rated. To make sure participants were committed to work hard on all activities, cinema vouchers were announced for the best overall performances. After signing consent, they were encouraged to leave all personal belongings (e.g., mobile phones) in the room before the experimenter led them to Room B where they were seated at a desk, Workstation 1.

Instruction phase The experimenter explained one by one the four activities at Workstation 1. During instructions she put activity-relevant objects in front of participants. The objects for each activity were stacked above each other ("mess up Workstation 1" in Fig. 1). For example, when explaining the "draw-a-person task," the experimenter dumped all crayons above a blank sheet of paper. Next, when explaining the "cartoon task" she opened the ring binder with cartoons and put it upon the crayons, and so forth. After all activities were explained the workstation was cleaned up. If participants were in the enactment condition, the experimenter instructed them where to put each object one at the time. If participants were in the observation condition, the experimenter similarly described each action verbally, but also carried out the actions herself. All objects were put back in their original place.

After the clearance of the first workstation, to adhere to the cover story, participants were asked to plan in which order and for how much of an 8-minute timeframe they wanted to work on each activity. It was stressed that beginning to work on all four activities was mandatory, but participants were encouraged to organize the time spent on each activity according 
to their strengths. Participants recorded the order in which they would start the activities as well as the amount of time they planned spending on each task (e.g., embroidery: 2 minutes) on a to-do-list which was subsequently pinned to the wall in front of the participant. During the testing phase, a timer was set to count backwards from 8 minutes. Thus, participants could check whether they were on their set schedule.

After having planned To-Do-List 1, the next step in the instruction phase involved that participants changed to a desk with a computer in order to practice the LDT. Partitions around this desk ensured that the objects mentioned in the LDT in Phase 2 were out of sight. The LDT was introduced as a vocabulary test, and they practiced the task in eight trials. The experimenter stressed the importance to change desks as fast as possible when time at Workstation 1 had expired, leaving all materials at the desk. In addition, a written reminder ("After eight minutes — change desks immediately!") was positioned next to the participant's To-Do-List 1.

For the final part of the instruction phase, participants were seated at another desk, Workstation 2. The procedure was identical to the first one: The four activities were explained one by one. Either the participant (enactment condition) or the experimenter (observation condition) cleaned up the workstation after all activities had been explained. Participants planned in which order and for how long they would work on these activities. To-Do-List 2 was pinned to the wall in front of them. Then, they were reminded to leave everything at the workstation and begin to work immediately on another LDT when time for the activities had expired. A written reminder to change desks immediately was put next to To-Do-List 2.

Activity phase If participants had no further questions, Phase 2 began. They were again seated at the first workstation where they worked on the activities as they had planned. The experimenter instructed participants to report to her in the room next door (Room A) to receive compensation once the experiment was over. Participants were asked to bring their used materials (e.g., rope with the knot) with them and were given the instruction, "Please prepare the desks as they are now before leaving the room." Thus, at this point participants formed the intention to clean up both workstations. To-be-replaced materials (e.g., a second embroidery frame) were clearly visible on a windowsill. The experimenter started the timer in front of the participant and left the room.

After 8 minutes, the timer gave a distinctive beep upon which the experimenter unexpectedly returned to Room B. She checked whether participants had changed to the chair in front of the computer and told them, "I have some time to clean up this workstation. Even so, I'd like to ask you to clean up the second workstation before leaving." Thus, prospective clean-up tasks of Workstation 1 were cancelled, but the clean- up tasks of Workstation 2 remained intention relevant. Across participants, each set of materials served equally often as tobe-maintained versus cancelled prospective clean-up tasks. Participants then answered some general questions about how they cope with time pressure, before starting the first LDT. These questions were included to give the experimenter some time to clean up the first workstation without disturbing participants during the LDT. Objects were returned to their previous place or replaced if necessary. All the materials that had been used were put into a box. The experimenter remained quietly in the room until participants had finished the LDT and had sat down at Workstation 2. The experimenter then started the timer again and left the room. After 8 minutes, participants completed another set of questions, and the second LDT that was introduced as "the final task." If participants were in the final LDT condition, critical words were presented in the final LDT (i.e., referring to the to-be maintained and the cancelled tasks). If participants were in the intermediate LDT condition, these words had appeared in the intermediate LDT. On completion, the words "The experiment is finished! Please inform the experimenter in the room next door" appeared on screen.

Memory tests At this point, we expected participants to carry out the prospective memory task without a further reminder. When participants reported to the experimenter in Room A, they completed a questionnaire including demographic data and an unexpected written free recall test of the prospective tasks. Finally, participants received compensation, were thanked, and debriefed. The experiment took about 45 minutes.

\section{Results}

For all statistical analyses, the Type I error was set at $\alpha=.05$. Two-tailed $p$ values are reported throughout, even though all hypotheses were directional.

\section{Prospective memory performance}

Experimenter protocols detailed which prospective clean-up tasks participants had realized before leaving the experimental room. Prospective memory performance was determined by the percentage of clean-up actions performed out of the total possible number (i.e., the number of activities that had been started). Actual percentages varied between .50 and 1 . Two participants had failed to start all four of the tasks because they spent too much time on one of the other tasks. Findings for each experimental condition are depicted in Table 1. As average scores for each encoding condition show, participants who enacted the clean-up tasks during instructions realized more tasks at the end of the experiment than participants who observed the experimenter carrying out the actions. This finding 
Table 1 Prospective memory performance (means and standard deviations) separately for each experiment and condition, and averaged

\begin{tabular}{|c|c|c|c|c|c|c|}
\hline \multirow[t]{2}{*}{ Encoding condition } & \multicolumn{2}{|c|}{ Enactment } & \multicolumn{2}{|c|}{ Observation } & \multicolumn{2}{|c|}{ Average } \\
\hline & $M$ & $(S D)$ & $M$ & $(S D)$ & $M$ & $(S D)$ \\
\hline \multicolumn{7}{|l|}{ Experiment 1} \\
\hline LDT: Intermediate & .88 & $(.16)$ & .82 & (.19) & .84 & $(.18)$ \\
\hline LDT: Final & .92 & (.14) & .85 & (.16) & .88 & $(.16)$ \\
\hline Average & .90 & $(.15)$ & .83 & $(.17)$ & .87 & $(.17)$ \\
\hline \multicolumn{7}{|l|}{ Experiment 2} \\
\hline With intention & .56 & $(.36)$ & .72 & $(.25)$ & .64 & $(.32)$ \\
\hline
\end{tabular}

contradicts Hypothesis 1. However, a 2 (encoding condition) $\times 2$ (critical LDT position) ANOVA revealed no significant effects of encoding condition, $F(1,47)<1.9, p>.18$ (both other $F_{\mathrm{S}}<1$ ). This lack of effect was probably due to a ceiling effect, which in turn also meant that the expected relationship between the intention-superiority effect and prospective memory performance could not be tested (see Hypothesis 5).

\section{Lexical-decision task}

In all experiments, incorrect trials and responses that differed from the participants' individual mean response by three standard deviations or more in the critical lexical-decision task were excluded from analyses (12\% in total). Mean response times for the cancelled and prospective task were computed, and the intention-superiority effect $\left(\mathrm{RT}_{\text {cancelled }}-\mathrm{RT}_{\text {prospective }}\right)$ was used as the main dependent variable (see Table 2). Positive values indicate an intention-superiority effect.
Reaction times to nonwords and noncritical words were not counterbalanced and thus were not evaluated.

A 2 (encoding) $\times 2$ (LDT position) ANOVA revealed a main effect of LDT position, $F(1,47)=20.47, p<.001, \eta_{p}$

$2^{2}=.30$, indicating a larger intention-superiority effect when the critical LDT was the final rather than the intermediate task, supporting Hypothesis 2. This effect was qualified by an Encoding Condition $\times$ LDT position interaction, $F(1,47)=$ $8.51, p=.005, \eta_{\mathrm{p}}{ }^{2}=.15$ (encoding main effect: $F<1$ ). We explored the interaction with simple main effect analyses. When the critical lexical-decision task was administered midexperiment, intention-superiority effects did not differ significantly between the enactment and observation condition (simple main effect: $F<2.3, p=.14$ ) and were close to zero. However, when the lexical-decision task was presented as the final task, we found a larger intention-superiority effect in the observation than in the enactment condition, $F(1,47)$ $=7.34, p=.009, \eta_{\mathrm{p}}{ }^{2}=.14$, in line with Hypothesis 3 .

\section{Retrospective memory}

Although we asked participants at free recall to explain the clean-up tasks in detail, they often wrote down general answers such as "prepare the second desk before leaving the room." Because of the missing details in recall protocols, we refrained from analyses of these data, not testing Hypothesis 4.

\section{Discussion}

In line with Hypotheses 2 and 3, we observed a smaller reaction-time difference between prospective and cancelled

Table 2 Average reaction times (ms, with standard deviations) for intentional-related stimuli and stimuli from the cancelled task and intentionsuperiority effects (ISE), separately for each experiment and condition, and averaged

\begin{tabular}{|c|c|c|c|c|c|c|c|c|c|c|c|c|c|c|}
\hline \multirow{3}{*}{$\begin{array}{l}\text { Encoding condition } \\
\text { Stimuli }\end{array}$} & \multicolumn{6}{|c|}{ Enactment } & \multicolumn{6}{|c|}{ Observation } & \multirow{2}{*}{\multicolumn{2}{|c|}{$\begin{array}{l}\text { Average } \\
\text { ISE }\end{array}$}} \\
\hline & \multicolumn{2}{|c|}{ Intention related } & \multicolumn{2}{|c|}{ Cancelled } & \multicolumn{2}{|c|}{ ISE } & \multicolumn{2}{|c|}{ Intention related } & \multicolumn{2}{|c|}{ Cancelled } & \multicolumn{2}{|c|}{ ISE } & & \\
\hline & $M$ & $(S D)$ & $M$ & $(S D)$ & $M$ & $(S D)$ & $M$ & $(S D)$ & $M$ & $(S D)$ & $M$ & $(S D)$ & $M$ & $(S D)$ \\
\hline \multicolumn{15}{|l|}{ Experiment 1} \\
\hline Intermediate task & 841 & $(142)$ & 844 & (143) & 4 & (12) & 862 & $(266)$ & 852 & $(255)$ & -9 & $(21)$ & -3 & (19) \\
\hline Final task & 865 & $(181)$ & 878 & (181) & 13 & (17) & 858 & $(160)$ & 886 & $(162)$ & 28 & (32) & 20 & (26) \\
\hline Average & 855 & (164) & 865 & $(165)$ & 9 & (16) & 860 & (209) & 871 & (204) & 11 & (33) & 10 & (26) \\
\hline \multicolumn{15}{|l|}{ Experiment 2} \\
\hline With intention & 936 & $(200)$ & 969 & $(218)$ & 33 & $(42)$ & 925 & $(146)$ & 986 & $(176)$ & 70 & (71) & 52 & (61) \\
\hline Without intention & 944 & $(212)$ & 954 & $(201)$ & 11 & $(51)$ & 974 & (135) & 970 & (148) & -3 & (80) & 3 & (67) \\
\hline Average & 939 & (203) & 962 & (208) & 23 & (47) & 948 & (142) & 979 & (162) & 36 & (83) & 30 & (68) \\
\hline
\end{tabular}

Note. In Experiment 2, all participants worked on the LDT as the final task of the experiment. For participant without intention formation, reaction times for intention-related stimuli refer to objects recently encountered at Workstation 2, control stimuli refer to objects presented earlier at Workstation 1 
tasks in the enactment condition than in the observation condition, but this intention-superiority effect was only observed if the lexical decision task was administered at the end of the experiment. It seems that our rather subtle instructions for the prospective tasks were sufficient to elicit intention-superiority effects in a lexical-decision task if these intentions were relevant in the near future. However, we could not test for the assumed relationship between intention-superiority effect and prospective memory performance because prospective memory performance was near ceiling. Therefore, we used more clean-up tasks in Experiment 2.

In line with Freeman and Ellis's (2003a, 2003b) action-superiority account we found smaller reactiontime advantages for intention-related materials after previous enactment compared to the observation condition. Interestingly, we found a smaller intention-superiority effect after enactment, although our control condition also drew attention to action-related information. Thus, self-performance versus other-performance appears to be the critical difference between the enactment and the control condition (and not whether attention is drawn to action-related information or not).

Replicating Schult and Steffens (2013), we found an intention-superiority effect if the lexical-decision task was associated with an opportunity to realize the intention in the near future. However, one could argue that the observed reaction-time advantage for stimuli associated with the prospective tasks also represents a reaction-time advantage for more recently presented objects. Yet if the observed effect were only due to priming, then we could have observed similarly large, but negative, effects for participants who worked on the critical LDT in the middle of the experiment. This was not the case. To further support our claim that the observed reaction-time advantage for intention-related materials in the LDT at the end of the experiment is more than priming for recently presented materials, we included a no-intention control condition in Experiment 2.

\section{Experiment 2}

The principal aim of Experiment 2 was to test the relationship between encoding condition, intention-superiority effect, and successful prospective memory. We tested whether the size of the intention-superiority effect mediated the relationship between encoding condition and prospective memory performance (see Hypothesis 5).

Hypothesis 2 now reads: There is an intentionsuperiority effect for materials associated with the prospective tasks as compared to the cancelled tasks if participants expect to enact the clean-up tasks after the lexical-decision task, but not if participants do not form an intention to clean up. The other hypotheses were identical to Experiment 1.

\section{Method}

\section{Participants and design}

We needed a larger sample than in Experiment 1 because we were mainly interested in the relation between the intention-superiority effect and prospective memory performance. The total sample consisted of 89 participants who took part in exchange for course credit or a small payment. The design was a 2 (encoding condition: enactment vs. observation, between subjects) $\times 2$ (intention formation: with vs. without, between subjects) $\times 2$ (word type: prospective list, cancelled list, within subject) factorial. We excluded six participants from analyses due to language problems or because they did not complete all test materials. Of the remaining 83 participants $\left(M_{\text {age }}=\right.$ 21.03 years, age range: $18-42$ years; $88 \%$ women), 45 formed an intention to clean up the desks at the end of the experiment $(n=22$ enacted the prospective task during instructions, $n=23$ watched the experimenter enacting the prospective task). The remaining 38 participants received no clean-up instructions $(n=18$ enacted the clean-up tasks during instruction, $n=20$ watched the experimenter). Given the increased sample size, the statistical power to detect a large interaction effect $(f=.40$; see J. Cohen, 1977) between encoding condition and intention formation in an ANOVA with $\alpha=.05$ was now 1 $-\beta=.95$ (Faul et al., 2007). For participants who formed an intention, the power to detect the expected correlation between intention-superiority effect and prospective memory performance $(r=.40)$ was close to $1-\beta=.90$ (if a one-tailed test were used).

\section{Materials and procedure}

Figure 2 illustrates the procedure of Experiment 2, which was carried out by several well-trained female experimenters unaware of the hypotheses. The general procedure was similar to Experiment 1 with the following exceptions. First, participants worked on six different activities at each of the two desks. Consequently, all participants enacted or observed $2 \times 6$ prospective clean-up tasks. To avoid material confounds, the activities and thus the respective prospective tasks were rotated across participants using a Latin square technique. We extended the time period available to work on each activity set to 10 minutes.

Second, only participants in the "with intention" condition were asked to clean up both desks before leaving the laboratory room. Participants in the control condition ("without intention") were simply asked to come to the 


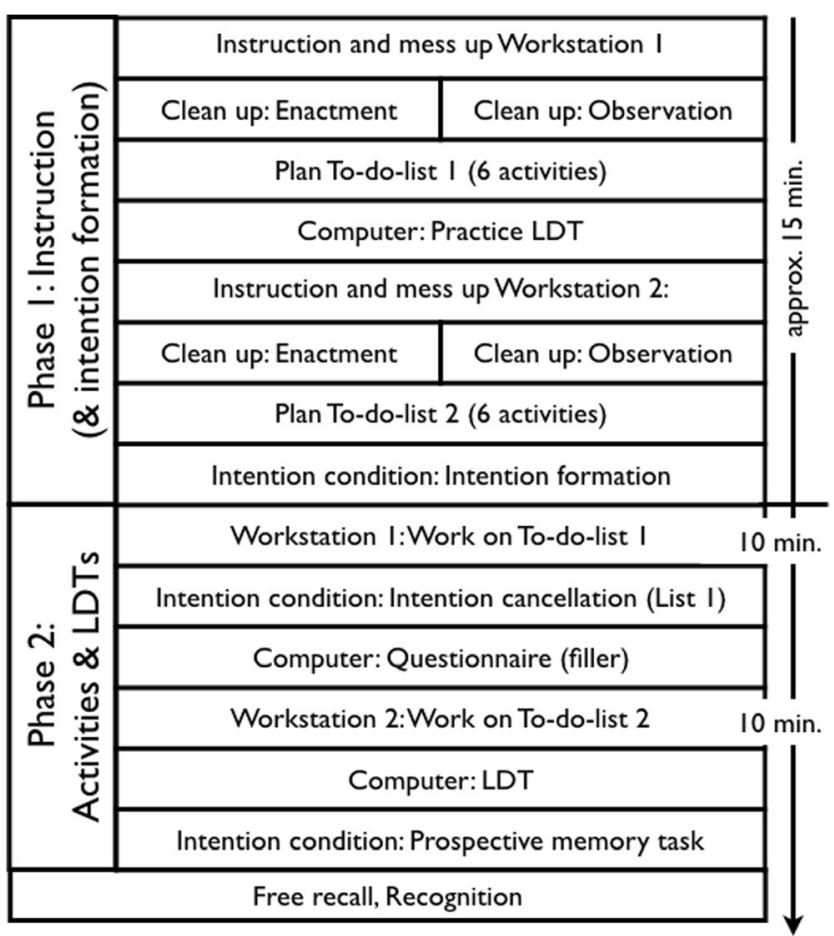

Fig. 2 Illustration of the procedure of Experiment 2

experimenter after the final task. Third, participants completed only one LDT. The LDT was always the last task of the experiment and consisted of 24 nouns related to the clean-up tasks (for participants "with intention," 12 nouns referred to the prospective tasks and 12 nouns to the cancelled ones), 24 new words, and 48 nonwords matched in word length. Between working on the first and second todo list, participants completed a short computer-based filler questionnaire. Similar to Experiment 1, they were instructed to answer this questionnaire immediately after the 10 minutes had expired. While they worked on the filler task, the experimenter cleaned up Workstation 1 and then left the room. To avoid the ceiling effect found in Experiment 1, the experimenter did not specifically remind them at this point that they still should clean up the second desk before leaving the room.

To avoid the global responses in free recall in Experiment 1, more specific instructions were given for free recall, and the experimenter checked the written free recall responses immediately and asked participants for details if they responded, for example, "clean up desk." After the free recall test, a recognition test sheet was handed to participants. Participants received a list describing 16 tidy-up tasks (the six prospective tasks, the six cancelled ones treated as false alarms in the analyses, and four new ones). They were asked to tick all tidy-up tasks they could have done before leaving the lab. We reminded them that some tidy-up tasks were already finished by the experimenter and should consequently not be ticked. The order of the tidy-up tasks was fixed across participants, but different tasks served as prospective and cancelled tasks for participants due to the Latin square technique.

\section{Results and discussion}

\section{Prospective memory}

Participants in the observation-intention condition tended to execute more clean-up tasks than participants in the enactment-intention condition, $F(1,43)=3.27, p=.078$, $\eta_{\mathrm{p}}{ }^{2}=.07$, providing some evidence for Hypothesis 1 (see Table 1).

\section{Lexical-decision task}

Incorrect trials and response times that differed from participants' individual mean response by three standard deviations or more in the lexical-decision task were excluded from analyses (in total 3\% of the critical words). Again, the main dependent variable was the difference between mean reaction times for stimuli related to clean-up actions at Workstation 1 (cancelled for participants in the intention condition) and mean reaction times for stimuli related to the clean-up actions at Workstation 2 (intention-relevant for participants in the intention condition). A 2 (encoding condition) $\times 2$ (intention formation) ANOVA revealed a main effect of intention formation, indicating an intention-superiority effect when participants formed an intention compared to participants with no intention, $F(1,79)=11.69, p=$ $.001, \eta p^{2}=.13$. This was consistent with Hypothesis 2. Although the predicted intention formation $\times$ encoding condition interaction missed the preset criterion of statistical significance, $F(1,79)=3.34, p=.071$, $\eta \mathrm{p}^{2}=.04$, we computed simple main effects testing for differences between encoding conditions. Replicating Experiment 1, for participants in the intention condition, the intention-superiority effect was smaller in the enactment encoding than in the observation condition, which would be statistically significant in a one-tailed test, $F(1,79)=3.80$, two-tailed $p=.055, \eta p^{2}=.05$, providing some evidence for Hypothesis 3. In the nointention condition, the reaction time difference was similarly small for participants in the enactment and the observation condition, $F<1$. Conversely, in the observation condition, the intention-superiority effect was larger in the intention condition than in the nointention condition, $F(1,79)=16.30, p<.001, \eta_{p}{ }^{2}$ $=.17$. Based on an action-superiority account, postulating similar memory representations for actions carried 
out, one would expect comparable effects for enactment encoding with and without intention. The data are compatible with this idea because there is no significant difference, $F(1,79)=1.39, p=.24$.

The cancelled task represents response times to stimuli associated with the activities completed at the first table about 10 minutes ago, whereas the response times to intentionrelevant stimuli were related to stimuli associated with activities executed a few minutes previously. Thus, in the nointention condition, a priming effect would result in faster responses for stimuli last encountered. Notably, the data showed no priming effect $(F<1)$.

\section{Retrospective memory}

In the intention condition, prompted free recall for the cleanup tasks was similar for participants in the enactment $(M=$ $.60)$ and the observation condition $(M=.63), F<1$, in line with Hypothesis 4. Regarding recognition, participants in the enactment condition $(M=.45)$ recognized fewer clean-up tasks correctly than participants in the observation condition $(M=.72), F(1,43)=6.56, p=.014, \eta_{\mathrm{p}}{ }^{2}=.13$. However, false alarm rates were also higher in the observation $(M=.33)$ than in the enactment condition $(M=.08), F(1,43)=7.88, p=$ $.007, \eta_{\mathrm{p}}{ }^{2}=.16$. When hits minus false alarms were analyzed (PR scores; see Snodgrass \& Corwin, 1988), there was no effect of encoding condition, $F<1$, not corroborating Hypothesis 4 for recognition.

\section{Mediation analysis}

Based on the regression approach suggested by Hayes (2013), we tested whether there is an indirect effect of encoding condition mediated by the intention-superiority effect on prospective memory performance. In other words, we tested whether data are in line with the idea that the increased accessibility of intention-related words mediates the effect of encoding condition on prospective memory performance. Recognition memory performance was included in the model as a potential alternative mediator variable (PR scores). Figure 3 shows the unstandardized regression coefficients. In a first regression model, we confirmed a statistically significant effect of encoding condition on the intentionsuperiority effect ( $t=2.09, p=.04)$. A second regression model showed a statistically significant effect of the intention-superiority effect on prospective memory performance $(t=5.08, p<.001)$. In this second model, the effect of encoding condition on prospective memory performance was not statistically significant $(|t|<1)$.

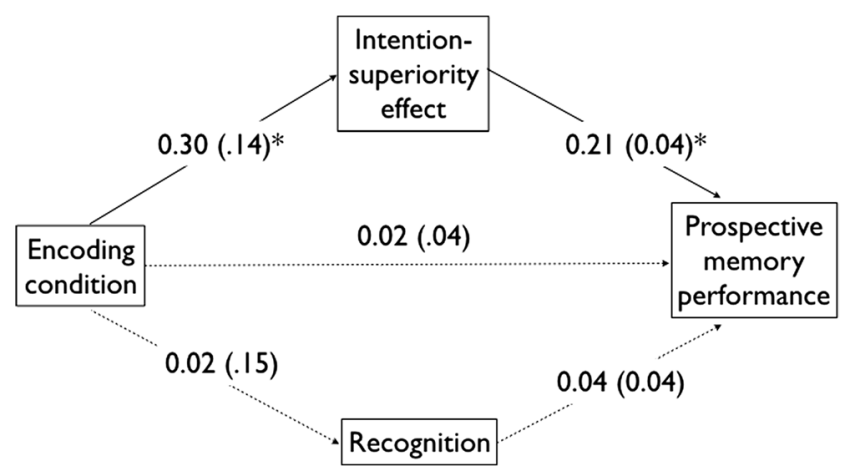

Fig. 3 Unstandardized regression coefficients (with standard errors) obtained in three regression models aimed at testing mediation: Models tested whether encoding condition affected (1) the intention-superiority effect, (2) recognition, and (3) whether intention-superiority effect, recognition, and encoding condition affected prospective memory performance. Dashed lines indicate nonsignificant effects

Bootstrapping analyses (with 10,000 resamples) demonstrated that the total indirect effect of encoding condition on performance mediated by the intention-superiority effect was statistically significant (i.e., the confidence interval did not span zero), $B=0.06$, Boot $S E=0.03,95 \%$ bias-corrected CI $[0.01,0.12]$ (completely standardized effect: $\beta=.20$ ). This finding is in line with the interpretation of full mediation of the effect of encoding condition on prospective memory performance via the intentionsuperiority effect. Recognition memory was nonsignificant as a mediator variable (indirect effect via PR scores: $B=0.0008$, Boot $S E=0.0096,95 \%$ bias-corrected CI [$0.01,0.03])$. The same analyses omitting recognition memory yielded the identical pattern of findings. Taken together, this experiment is the first to demonstrate a functional relationship between facilitated processing of intention-related information and successful prospective memory performance in the postponed-intention paradigm.

\section{General discussion}

The central aim of this research was to test under relatively realistic conditions whether there is a functional relationship between the activation of intentional-relevant words (i.e., the intention-superiority effect) and prospective memory. At the same time, we tested effects of encoding condition (enactment vs. observation) on memory activation and performance. Our main findings can be summarized as follows. The intentionsuperiority effect was smaller in the enactment condition than in the observation condition (significantly so in Exp. 1, two- 
tailed $p<.06$ in Exp. 2). Using a mediation model, we found evidence of the following prediction: The more a participant demonstrated activation of intention-relevant words, the better was his or her prospective memory performance. This indicates a functional relationship between memory activation and memory performance (Exp. 2). These data suggest that participants in the enactment condition showed worse prospective memory performance than those in the observation condition because they showed less activation of intention-relevant words (in Exp. 2). Concurrently, we replicated the context sensitivity of the intention-superiority effect (Exp. 1), and we excluded a simple priming explanation for the time pattern of activation (Exp. 2). Also, the intention-superiority effect was descriptively larger after enactment with than without intention, but as this finding was not statistically significant, it will not be discussed further: It is ambiguous how these data line up with the action-superiority account (Freeman \& Ellis, 2003b).

The observed relationship between intentionsuperiority effects and prospective remembering generally supports Goschke and Kuhl's (1993) assumption that a heightened accessibility of intention-related stimuli during the delay interval supports the realization of intended tasks. In contrast to their model of increased accessibility following encoding, our data support the notion of a context-sensitive intention-superiority effect that is only observable if there are proximal opportunities to execute an intention (replicating Schult \& Steffens, 2013). In both of the present studies, participants were primed to think that the lexical-decision task would be the "last task." This may have facilitated preparatory processes for tasks intended next - that is, the clean-up tasks. These findings are in line with several past studies that support increased accessibility of intention-related materials (Meilán, Carro, Arana, \& Pérez, 2011; Van Kerckhove, Geuens, \& Vermeir, 2012), including words associated with the retrieval opportunity (Förster et al., 2005). Our results are also consistent with Goschke and Kuhl's (1993) own findings of an intention-superiority effect in the last block of a recognition task (i.e., immediately before participants expected to carry out the intention), but not in the preceding block.

We should discuss similarities and differences between the present findings and those on the enactment effect in retrospective memory. The finding of better recall and recognition after enacting simple action phrases (e.g. "throw a ball") compared to verbal learning is a well-established phenomenon of retrospective memory (e.g., see Engelkamp, 1998; Zimmer et al., 2001, for overviews). This retention advantage seems to last for several days (e.g., Carroll et al., 1999; Hornstein \&
Mulligan, 2004; Kubik, Söderlund, Nilsson, \& Jönsson, 2014). However, our participants enacted or observed the tasks during encoding, and findings regarding differences between enactment and observation at encoding are less clear (see Steffens et al., 2015). Thus, finding few differences in prompted retrospective free recall between encoding conditions in Experiment 2 is not surprising. It is less expected that corrected recognition scores did not differ between encoding conditions. Typically, enactment leads to better recognition than observation (Schult, von Stülpnagel, \& Steffens, 2014). We speculate that the small number of stimuli could be a reason why the typical enactment effect was not replicated. According to previous research, the enactment effect depends on the specific stimuli used (R. L. Cohen, Peterson, \& Mantini Atkinson, 1987).

Of further interest, after enactment compared to observation, prospective memory performance was impaired in Experiment 2 (Experiment 1 yielded a ceiling effect, so we refrain from interpreting the descriptive reversed pattern found there). According to Freeman and Ellis (2003a, 2003 b) we should have found similarly small intentionsuperiority effects for both encoding conditions because all participants intended to enact the tasks later, so that it should not affect the outcome whether participants encoded by enactment or by another encoding format. We are not sure why the present pattern of findings was different from theirs. Perhaps participants in the enactment condition did not encode retrieval opportunity and intended action as well as participants in the observation condition. If so, this could be explained by the notion that carrying out actions is accompanied by an internal focus of attention and thus actions are less related to the encoding context (Fischer, Schult, \& Steffens, 2015; Gopie \& MacLeod, 2009). However, encoding of the intended tasks and instructions for the prospective tasks were separated in the present experiments, which makes this explanation less likely. Alternatively, participants in the enactment condition could have inadequately judged their ability to remember to perform the tasks later. Recent research suggests that enactment may appear deceptively easy (von Stülpnagel et al., 2016). However, we have no further data to support this notion. A smaller intention-superiority effect has previously been reported after successful task completion (Marsh et al., 1998). Possibly, the enactment encoding condition shared features with successful task completion, so that the accessibility of intention-related words was lowered.

Importantly, it has previously been suggested that the intention-superiority effect reflects strategic rehearsal processes (Altmann \& Trafton, 2002). Our cover story 
was intended to make the prospective memory task as inconspicuous as possible to minimize the likelihood of rehearsal. In spite of our subtle instructions, reliable intention-superiority effects were observed, speaking against that explanation.

Marsh et al. (1998) noted that the intention-superiority effect could either be due to a heightened accessibility of intention-related information, or to an inhibition of irrelevant information (i.e., the cancelled list), or to a combination of both. Indeed, the reaction times in the no-intention control condition included in Experiment 2 suggest that both activation and inhibition may contribute to the intention-superiority effect (see Table 2).

As we mentioned in the theoretical introduction, Schaefer and colleagues (1998) suggested several explanations why prospective memory could be impaired after enactment encoding. The present research was not designed to test these explanations, and none of them can be excluded on the basis of the present findings. Still, we showed that compared to the observation condition, the smaller intention-superiority effect after enactment encoding is related to worse prospective memory performance after enactment encoding. This pattern of findings supports our reasoning that high accessibility of intention-relevant information is important for prospective memory performance.

As is the case for most research in the postponed intention paradigm, two limitations of the present study relate to the fact that only a limited set of materials could be investigated (i.e., 12 activities at most; see Exp. 2), and that intentions were postponed only for a short time period. The activation pattern of long-term goals that may be postponed for years or even decades cannot be investigated (e.g., "Once I have enough money, I will buy a holiday home" or "If I ever go to Dresden again, I will use the leftover bus ticket from last year"). A relatively more realistic, but less controlled, paradigm infers the accessibility of intentions by comparing everyday retrospective and prospective memory (e.g., Penningroth, 2005). For example, during a speeded retrieval task, participants list what they have done last week (retrospective), and what they intend to do next week (prospective). Higher numbers of prospective than retrospective tasks indicate an intention-superiority effect. Because of the differences between paradigms, however, findings cannot be compared directly. As one example, for female, but not male participants, Penningroth (2005) found an intention-superiority effect in the most accessible memories. The small number of men who participated in our experiments makes it impossible to explore gender differences. Freeman and Ellis (2003c) modified the described paradigm by asking participants 1 week after the prospective task to list the tasks they had done the previous week. Thus, the prospective and the retrospective tasks concerned the same pool of intentions. Younger adults who carried out many tasks during the critical week that they had failed to report in the prospective task also completed fewer of the intentions that they had reported in the prospective task. Our findings provide a conceptual replication of that pattern that also indicates a functional relationship between intention accessibility and prospective memory performance. However, Freeman and Ellis found no such negative correlation for older adults. Such individual differences in the intention-superiority effect suggest that the relationship between the accessibility of intention-related materials and prospective memory performance depends on moderating factors that need to be tested in future research.

To rule out the idea that the intention-superiority effect is simply a priming effect for recently presented materials, participants in the control group in Experiment 2 were not instructed to clean up at the end. As they did not show an intention-superiority effect, we conclude that the intentionsuperiority effect we observed in Experiment 1 is more than simply a priming effect for recently presented materials. However, one could argue that some participants expected some sort of future activity with the objects (i.e., cleaning up), others did not, so there was still a difference between conditions, which is another limitation of the present research. To rule out this idea, future research could instruct participants in the control condition that a memory test would follow. A final limitation is that not all of our findings were as strong as expected, and some would be statistically significant only if one-tailed tests were used. Still, the overall pattern across the two experiments suggests that the main findings are replicable. However, effect sizes may be smaller than originally expected.

To conclude, researchers have hypothesized that the increased accessibility of intention-relevant information in the postponed intention paradigm is functional for good prospective memory performance. The present research is the first to provide the critical evidence of a link between both. Thus, if "milk" pops into your memory toward the end of a long work day, this will help you remember to stock your fridge.

Author note The current research was funded by grants from the German Science Foundation to the second author (Ste 938/7-1, Ste 938/ 7-2). We thank Marcel Schmitt and Anders Sønderlund for very valuable comments on a previous version of this article. 


\title{
Appendix
}

Table 3 Materials used in Experiments 1 and 2

\author{
Task description
}

English translations of words used Original German words used in the LDT in the LDT

Experiment 1

To-do-list 1:

1. Invent headlines for cartoons in a ring binder (placed on the windowsill) * Ring binder, windowsill

2. Draw a person (with crayons from a tin box)* crayons, tin box

3. Find the missing element in drawings (that are in a folder) *

drawing, folder

4. Embroider a face (with a needle and sewing thread)

To-do-list 2:

1. Sort picture cards in envelopes so that a logical order results *

2. Use small geometrical forms (from a wooden box) to create larger geometrical forms (Tangram game)

3. Use a napkin to fold a boot from a folding instruction

4. Draw ways through labyrinths with a pencil just sharpened

Experiment 2

1. Put marbles into a jar whenever you hear a signal

2. Draw a mandala (with a circle from a pencil case)

3. Use a text marker to highlight the names of plants hidden in a text

4. Translate words using dictionaries (that are in a box)

5. Use all same-colored puzzle pieces (from a cloth bag) to create figures

6. Craft a raft with corks and rubber bands from a wooden box

7. Tie a specific knot using an instruction

8. Use small plates to create specific patterns needle, sewing thread

Cards, envelope

puzzle pieces, wooden box

napkin, folding instruction

pencil, sharpener

jar, marbles

circle, pencil case

text marker, rubber band

dictionaries, box

puzzle pieces, cloth bag

materials, wooden box

rope, instruction

small plates, box
Ringblock, Fensterbrett

Buntstifte, Metallbox

Bildvorlage, Ordner

Nadel, Nähgarn

Kärtchen, Umschlag,

Puzzleteile, Holzschachtel

Serviette, Bastelanleitung

Bleistift, Anspitzer

Becher, Murmel

Zirkel, Federtasche

Textmarker, Gummiband

Wörterbücher, Karton

Puzzleteile, Stoffbeutel

Material, Holzschachtel

Seil, Anleitung

Plättchen, Kasten

Note. * Tasks also used in Experiment 2. In Experiment 1, to-do lists were counterbalanced (i.e., List 1 was List 2 for half of the participants). In Experiment 2, activities were counterbalanced across lists using a Latin square design

\section{References}

Altmann, E. M., \& Trafton, J. G. (2002). Memory for goals: An activation-based model. Cognitive Science, 26, 39-83.

Carroll, M., Mazzoni, G., Andrews, S., \& Pocock, P. (1999). Monitoring the future: Object and source memory for real and imagined events. Applied Cognitive Psychology, 13, 373-390.

Cohen, J. (1977). Statistical power analysis for the behavioral sciences (Revth ed.). Hillsdale: Erlbaum.

Cohen, R. L., Peterson, M., \& Mantini Atkinson, T. (1987). Interevent differences in event memory: Why are some events more recallable than others? Memory and Cognition, 15, 109-118.

Deutscher Wortschatz [Database]. (2016). Retrieved from http://wortschatz.uni-leipzig.de/index.html

Dockree, P. M., \& Ellis, J. A. (2001). Forming and canceling everyday intentions: Implications for prospective remembering. Memory \& Cognition, 29, 1139-1145.

Ellis, J. A. (1996). Prospective memory or the realization of delayed intentions: A conceptual framework for research. In M. Brandimonte, G. O. Einstein, \& M. A. McDaniel (Eds.), Prospective memory: Theory and application (pp. 1-22). Mahwah: Erlbaum
Engelkamp, J. (1998). Memory for actions. Hove: Psychology Press

Faul, F., Erdfelder, E., Lang, A.-G., \& Buchner, A. (2007). G*power 3: A flexible statistical power analysis program for the social, behavioral, and biomedical sciences. Behavior Research Methods, 39, 175-191.

Fischer, N., Schult, J. C., \& Steffens, M. C. (2015). Source and destination memory in face-to-face interaction: A multinomial modeling approach. Journal of Experimental Psychology: Applied, 21, 195-204.

Förster, J., Liberman, N., \& Higgins, E. T. (2005). Accessibility from active and fulfilled goals. Journal of Experimental Social Psychology, 41, 220-239.

Freeman, J. E., \& Ellis, J. A. (2003a). Aging and the accessibility of performed and to-be-performed actions. Aging, Neuropsychology, and Cognition, 10, 298-309.

Freeman, J. E., \& Ellis, J. A. (2003b). The representation of delayed intentions: A prospective subject-performed task? Journal of Experimental Psychology: Learning, Memory, and Cognition, 29, 976-992.

Freeman, J. E., \& Ellis, J. A. (2003c). The intention-superiority effect for naturally occurring activities: The role of intention accessibility in everyday prospective remembering in young 
and older adults. International Journal of Psychology, 38, 215-228.

Garry, M., Manning, C. G., Loftus, E. F., \& Sherman, S. J. (1996). Imagination inflation: Imagining a childhood event inflates confidence that it occurred. Psychonomic Bulletin \& Review, 3, 208-214.

Gilbert, S., Hadjipavlou, N., \& Raoelison, M. (2013). Automaticity and control in prospective memory: A computational model. PLoS ONE, 8, e59852.

Gopie, N., \& MacLeod, C. M. (2009). Destination memory: Stop me if I've told you this before. Psychological Science, 20, 1492-1499.

Goschke, T., \& Kuhl, J. (1993). Representation of intentions: Persisting activation in memory. Journal of Experimental Psychology: Learning, Memory, and Cognition, 19, 1211-1226.

Graf, P., \& Uttl, B. (2001). Prospective memory: A new focus for research. Consciousness and Cognition, 10, 437-450.

Hayes, A. F. (2013). Introduction to mediation, moderation, and conditional process analysis: A regression-based approach. New York: Guilford Press.

Horn, S. S., Bayen, U. J., Smith, R. E., \& Boywitt, C. D. (2011). The multinomial model of prospective memory: Validity of ongoing-task parameters. Experimental Psychology, 58, 247-255.

Hornstein, S. L., \& Mulligan, N. W. (2004). Memory for actions: Enactment and source memory. Psychonomic Bulletin \& Review, $11,367-372$.

Koriat, A., Ben-Zur, H., \& Nussbaum, A. (1990). Encoding information for future action: Memory for to-be-performed tasks versus memory for to-be-recalled tasks. Memory \& Cognition, 18, 568-578.

Kubik, V., Söderlund, H., Nilsson, L.-G., \& Jönsson, F. U. (2014). Individual and combined effects of enactment and testing on memory for action phrases. Experimental Psychology, 61, $347-355$.

Kvavilashvili, L., \& Fisher, L. (2007). Is time-based prospective remembering mediated by self-initiated rehearsals? Role of incidental cues, ongoing activity, age, and motivation. Journal of Experimental Psychology: General, 136, 112-132.

Lebiere, C., \& Lee, F. J. (2002). Intention superiority effect: A contextswitching account. Cognitive Systems Research, 3, 57-65.

Lee, J. H., \& McDaniel, M. A. (2013). Discrepancy-plus-search processes in prospective memory retrieval. Memory \& Cognition, $41,443-451$

Lewin, K. (1926). Vorsatz, wille und bedürfnis [Intention, will, and need]. Psychological Research, 7, 330-385.

Manzi, A., \& Nigro, G. (2008). Long-term memory for performed and observed actions: Retrieval awareness and source monitoring. Memory, 16, 595-603.

Marsh, R. L., Hicks, J. L., \& Bink, M. L. (1998). Activation of completed, uncompleted, and partially completed intentions. Journal of Experimental Psychology: Learning, Memory, and Cognition, 24, 336-349.

Marsh, R. L., Hicks, J. L., \& Bryan, E. S. (1999). The activation of unrelated and canceled intentions. Memory \& Cognition, 27, 320-327.

McDaniel, M. A., Guynn, M. J., Einstein, G. O., \& Breneiser, J. (2004). Cue-focused and reflexive-associative processes in prospective memory retrieval. Journal of Experimental Psychology: Learning, Memory, and Cognition, 30, 605-614.
McDaniel, M. A., \& Scullin, M. K. (2010). Implementation intention encoding does not automatize prospective memory responding. Memory \& Cognition, 38, 221-232.

Meilán, J. J. G., Carro, J., Arana, J. M., \& Pérez, E. (2011). Intention superiority effect on implicit memory of shopping lists: Activation and inhibition processes. Motivation and Emotion, 35, 144-150.

Penningroth, S. L. (2005). Free recall of everyday retrospective and prospective memories: The intention-superiority effect is moderated by action versus state orientation and by gender. Memory, 13, 711-724.

Penningroth, S. L. (2011). When does the intention-superiority effect occur? Activation patterns before and after task completion, and moderating variables. Journal of Cognitive Psychology, 23, $140-156$.

Penningroth, S. L., Graf, P., \& Gray, J. M. (2012). The effect of a working memory load on the intention-superiority effect: Examining three features of automaticity. Applied Cognitive Psychology, 26, 441-450.

Pereira, A., Ellis, J., \& Freeman, J. (2012a). Is prospective memory enhanced by cue-action semantic relatedness and enactment at encoding? Consciousness and Cognition: An International Journal, 21, 1257-1266.

Pereira, A., Ellis, J. A., \& Freeman, J. E. (2012b). The effect of age, enactment, and cue-action relatedness on memory for intentions in the virtual week task. Aging, Neuropsychology, and Cognition, 19, $549-565$.

Schaefer, E. G., Kozak, M. V., \& Sagness, K. (1998). The role of enactment in prospective remembering. Memory \& Cognition, 26, 644-650.

Schult, J. C., \& Steffens, M. C. (2011). On the representation of intentions: Do personally relevant consequences determine activation? Memory \& Cognition, 39, 1487-1495.

Schult, J. C., \& Steffens, M. C. (2013). Tuned for the future: Intentions are only accessible when a retrieval opportunity is near. Memory \& Cognition, 41, 1252-1260.

Schult, J. C., von Stülpnagel, R., \& Steffens, M. C. (2014). Enactment versus observation: Item-specific and relational processing in goal-directed action sequences (and lists of single actions). PLOS ONE, 9, e99985.

Snodgrass, J. G., \& Corwin, J. (1988). Pragmatics of measuring recognition memory: Applications to dementia and amnesia. Journal of Experimental Psychology: General, 117, 34-50.

Steffens, M. C., von Stülpnagel, R., \& Schult, J. C. (2015). Memory recall after "learning by doing" and "learning by viewing": Boundary conditions of an enactment benefit. Frontiers in Psychology, 6, 1907.

Van Kerckhove, A., Geuens, M., \& Vermeir, I. (2012). Intention superiority perspectives on preference-decision consistency. Journal of Business Research, 65, 692-700.

von Stülpnagel, R., Schult, J., Richter, C., \& Steffens, M. C. (2016). Cognitive costs of encoding novel natural activities: Can "learning by doing" be distractive and deceptive? The Quarterly Journal of Experimental Psychology, 69, 1545-1563.

Zimmer, H. D., Cohen, R. L., Guynn, M. J., Engelkamp, J., Kormi Nouri, R., \& Foley, M. A. (2001). Memory for action: A distinct form of episodic memory? New York: Oxford University Press. 